\title{
APRECIACIÓN DEL HUMOR Y DIMENSIONES BÁSICAS DE PERSONALIDAD: EVIDENCIAS EXTERNAS DE VALIDEZ DE LA ESCALA DE APRECIACIÓN DEL HUMOR, EAHU
}

\author{
H. Carretero-Dios \\ C. Pérez \\ G. Buela-Casal
}

Facultad de Psicología. Universidad de Granada.

\section{RESUMEN}

El objetivo principal de este estudio ha sido obtener evidencias externas de validez de la Escala de Apreciación del Humor, EAHU. Para esto se analiza la relación entre las puntuaciones obtenidas con esta escala y las dimensiones básicas de personalidad del modelo PEN de Eysenck, y el NEO de McCrae y Costa. La muestra del estudio fue de 323 participantes con edades comprendidas entre los 18 y 76 años (177 hombres y 146 mujeres).

En los resultados obtenidos puede observarse que los aspectos emocionales de la apreciación del humor (respuestas de diversión y rechazo hacia el material humorístico) estarían en general representados por los factores extraversión $y$ neuroticismo. Mientras, los aspectos cognitivos (humor con incongruencia-resolución frente a sin sentido) se verían especialmente relacionados con el factor de apertura a la experiencia. Por último, las categorías relacionadas con los componentes motivacionales de la apreciación del humor (humor 
negro, sexualy de denigración sexual), estarían más relacionadas con los factores responsabilidad y amabilidad, siendo la extraversión un importante factor predictor del humor sexual. Aunque los datos encontrados son coincidentes con las predicciones efectuadas, las correlaciones y coeficientes de regresión suelen ser bajos.

Palabras clave: SENTIDO DEL HUMOR; APRECIACIÓN DEL HUMOR;

PERSONALIDAD; ESCALA DE APRECIACIÓN DEL HUMOR; EAHU.

\section{SUMMARY}

To obtain external validity evidences of EAHU scale is the aim of this study. Relationships between EAHU scale scores and basic personality dimensions provided by Eysenck's PEN model and Costa and MCRae's NEO model was analyzed with 323 participants aged from 18 to 76 (177 males and 146 females).

Results indicated that while emotional components of humor appreciation (funniness and aversiveness answers) were related to extraversion and neuroticism, cognitive components (incongruity-resolution humor and nonsense humor) were specially related to openness to experience. Finally, motivational aspects of humor appreciation (black humor, sexual humor, and men and women disparagement humor) were related to agreeableness and conscientiousness factors, being extraversion predictor of sexual humor.

Data were on line with predictions, but correlations and regression coefficients were low and that fact was considered and discussed taking into account Pelechano's theoretical model: El modelo de Parámetros

Key wOrdS: SENSE OF HUMOR; HUMOR APPRECIATION; PERSONALITY; HUMOR APPRECIATION SCALE; EAHU. 


\section{INTRODUCCIÓN}

Dentro de la psicología, aunque tarde y quizá aún minoritariamente, parece existir un movimiento hacia un "paradigma de la complejidad", el cual rige desde hace tiempo otras disciplinas científicas. Como otros autores han señalado recientemente (Pelechano y Rivero, 2004), en las últimas décadas de la investigación en psicología puede observarse una vocación de complejidad, caracterizada, entre otras cosas, por la aceptación de temas de estudio que hasta no hace demasiado tiempo eran obviados como objeto de investigación. Ejemplos de esos temas serían la felicidad, el amor o la sabiduría, ocupando la investigación del sentido del humor un lugar destacado, desde hace aproximadamente tres décadas, en este surgimiento del estudio de lo complejo asociado a la llamada "psicología positiva" (Lefcourt, 2002).

A pesar de no existir un acuerdo sobre la definición del sentido del humor y sobre los componentes que lo integran (Driessen, 2001; Roeckelein, 2002; Wycoff, y Prior, 2003), se podría afirmar que la dimensión sobre la que puede observarse un mayor consenso, en cuanto a catalogarla como faceta esencial del sentido del humor, es la apreciación del humor (Norrick, 2003), la cual hace referencia al grado de diversión que se experimenta como respuesta ante distintos estímulos humoristicos (chistes, situaciones, etc.).

En el campo de trabajo de la apreciación del humor, uno de los temas de estudio clásicos ha sido el de situar a este constructo en un espacio de dimensiones básicas de personalidad. El análisis de la relación entre dimensiones básicas de personalidad y apreciación del humor, no obstante, adolece generalmente de problemas metodológicos serios. Así, para estos estudios se suelen usar instrumentos elaborados para el trabajo en cuestión, sin una estructura metodológica rigurosa para el desarrollo de éstos, y donde las características psicométricas básicas se desconocen. Las escalas consisten en un grupo de chistes o viñetas seleccionadas por el autor principal en función del aspecto que se supone recogen (humor sexual, agresivo, etc.,), pero que no son sometidos a análisis empíricos que revelen la estructura subyacente a ese grupo de chistes.

Aunque lo comentado suele ser la norma, la notable excepción la aporta el grupo de Willibald Ruch (para una revisión véase Ruch, 1992; 
Ruch y Hehl, 1998). A través de numerosos estudios, fundamentalmente basados en análisis factoriales, usando muestras diversas, con edades diferentes (Ruch, McGhee, y Hehl, 1990), nacionalidades distintas (Forabosco y Ruch, 1994), etc., han llegado a consolidar una propuesta para la apreciación del humor, que proporciona una herramienta de evaluación; el 3 WD humor test (del alemán Witz-Dimensionen). Este instrumento sirve para diferenciar tres dimensiones, una de contenido (humor sexual) y dos relacionadas con los procesos cognitivos que pueden aislarse como respuesta a la estructura interna de los chistes, viñetas, etc. (humor con incongruencia-resolución y humor sin sentido). Para evaluar a los participantes, se considera la diversión y aversión experimentada hacia el material humorístico recogido en la escala, teniendo en cuenta pues, tanto respuestas de valencia positiva (diversión) como negativa (aversión), aportación exclusiva de este grupo de trabajo dentro del campo de estudio de la apreciación del humor.

Siguiendo la propuesta integradora de Martin (1998), basada en los posicionamientos de Eysenck (1942), se acepta separar las aportaciones de los grandes sistemas de personalidad en función de que los resultados sirvan para "capturar" los aspectos emocionales, cognitivos y motivacionales de la apreciación del humor.

Para los aspectos emocionales, los factores de personalidad de referencia serían la Extraversión (estrechamente vinculada a un estado de ánimo positivo) y el Neuroticismo (como factor asociado a la inestabilidad emocional, ansiedad, depresión, etc.). Estos dos factores deberían verse relacionados fundamentalmente con, para el caso de la Extraversión, las respuestas de valencia positiva de la apreciación del humor (diversión ante chistes y viñetas), y para el Neuroticismo, con las respuestas de valencia negativa (aversión hacia el material humoristico).

Aunque la lógica que subyace a estas predicciones se fundamenta teóricamente, los hallazgos, tanto teniendo como referencia al sistema PEN de Eysenck, como el de los cinco grandes de McCrae y Costa (1999), y usando el 3 WD del grupo de Ruch, ponen de manifiesto que las correlaciones entre Extraversión y diversión, aunque suelen ser positivas, raramente alcanzan la significación estadística (Ruch, 1992; Ruch y Hehl, 1998). De igual forma, el Neuroticismo aparece 
correlacionado positivamente con las respuestas de valencia negativa de la apreciación del humor, aunque la significación estadística es la excepción más que la regla.

A pesar de la falta de significación estadística para la Extraversión y el Neuroticismo, la relevancia de estos dos factores, y dada la tendencia de las correlaciones, no se debe descartar. Cuando se trabajan con dimensiones no básicas de personalidad, pero relacionadas con los factores generales de Neuroticismo y Extraversión, las correlaciones aparecen con significación estadística en la dirección esperada (Ruch y Carrell, 1998). Así, los valores de correlación entre Neuroticismo, Extraversión y apreciación del humor son normales al tratarse la apreciación de un chiste o vin̄eta de una respuesta mucho menos estable y más dependiente de eventos contextuales que la de los factores básicos de personalidad considerados. Esto hace que la predicción sea leve (Pelechano, 2000), siendo la dirección de las correlaciones un apoyo a los planteamientos.

En lo que respecta a los aspectos cognitivos, aqui el interés se centraria en dos de las dimensiones propuestas por Ruch (Ruch y Hehl, 1998): humor con incongruencia-resolución y humor sin sentido. Estas dos categorías de humor tendrían su origen en Aristóteles, al manifestar en su Retórica que una forma de que sus asistentes a las tertulias rieran era crear una fuerte expectativa de lo que iba a decir, para luego sorprenderles con algo que ellos no esperaban, algo incongruente. Aquí, la distinción entre humor con incongruenciaresolución frente a humor sin sentido, estaria en el grado en el que la paradoja o incongruencia observada, acaba cobrando sentido para el receptor.

En el humor sin sentido, la incongruencia por sí sola sería una condición suficiente para provocar una respuesta de humor. En el humor con incongruencia-resolución, podria observarse un proceso con dos etapas, la percepción de la incongruencia y la resolución total de ésta (Forabosco, 1992). Para explicar este planteamiento, se adapta de Forabosco (1992) el siguiente chiste:

"Una joven pareja de chinos está de viaje de luna de miel recorriendo en tren toda América. Un pasajero situado junto a ellos, entabla una conversación con el hombre. En un determinado momento, este pasajero le pregunta: "Sé que tenéis muy pocas libertades 
en vuestro pais. Por ejemplo, ¿cuándo fueron sus últimas elecciones?". A lo que el chino contesta, "justo antes de la melienda".

Los postulados de la incongruencia-resolución se aplican al chiste de la siguiente manera. La respuesta del chino es incongruente a la pregunta de su compañero de viaje. Las elecciones (políticas) no se hacen antes de la merienda en un tren de viajeros. La siguiente fase, la resolución, supone encontrar una regla cognitiva que solucione la incongruencia. Para el chiste presentado, la regla es la no distinción en el idioma chino, entre los fonemas $/ / / \mathrm{y} / \mathrm{r} /$. "Cuando elecciones es comprendido como erecciones, el chiste toma sentido" Forabosco (1992; p. 48.).

Para estudiar la relación entra dimensiones básicas de personalidad y la diversión y aversión hacia el humor con incongruencia-resolución y humor sin sentido (con incongruencia sin completa resolución), los factores de personalidad a considerar serían los que están más relacionados igualmente con aspectos cognitivos. Para el caso del FFM y usando el 3 WD (Ruch y Hehl, 1998), se obtienen resultados que ponen de manifiesto que el factor de Apertura a la Experiencia es una dimensión a tener en cuenta. La Apertura a la Experiencia recoge elementos tales como la curiosidad, imaginación, inteligencia y creatividad. Los resultados encontrados usando este factor se resumirían diciendo que los participantes con puntuaciones altas en Apertura a la Experiencia se divierten más y rechazan menos el humor sin sentido.

La dimensión Psicoticismo de la propuesta de Eysenck, donde algunas de sus facetas recogen componentes de creatividad, también podría ser un factor a considerar para los aspectos cognitivos de la apreciación del humor, en cuanto a una preferencia mayor por el humor más complejo (humor sin sentido), aunque para este caso los datos son menos concluyentes (Ruch, 1999).

Ruch (1994), apuntó que la dimensión Psicoticismo sería un factor que principalmente serviria para "recoger" una tendencia hacia un determinado material humorístico u otro en función del contenido que sirva para definirlo, es decir, sería un factor relevante para estudiar los aspectos motivacionales. En este sentido, habría que decir que la relación entre Psicoticismo y diversión y aversión hacia la categoría de humor sexual de la propuesta de Ruch ha sido inexistente. Sin 
embargo, se postula que si se trabajara con escalas con contenidos distintos, y más cercanos conceptualmente a los elementos que sirven para definir a este factor de personalidad (humor negro), las correlaciones deberian emerger.

La afirmación anterior se vería apoyada con los datos obtenidos para el factor Extraversión. Aunque como ya se ha dicho, éste tendría su papel principal para "capturar" las respuestas de valencia positiva hacia el humor en general, sus componentes operativos deberian servir para aislar igualmente preferencias por un contenido específico, en concreto el sexual. Así, se ha constatado como la extraversión se relaciona de manera positiva con la diversión hacia el humor sexual (Martin, 1998).

Para recoger los aspectos motivacionales de la apreciación del humor, es sumamente importante contar con escalas que recojan factores diversos en cuanto al contenido. Sin embargo, las pruebas adolecen de los problemas comentados, y el instrumento de referencia, el 3 WD, sólo se ocupa de la temática sexual. Contenidos humorísticos centrados en grandes tragedias, catástrofes, etc. (humor negro), o en ridiculizar a los demás (humor de denigración), los cuales reflejarían marcos teóricos clásicos, no tienen su correspondencia en escalas psicométricamente avaladas, lo que afecta a los datos empíricos que hasta la fecha se han obtenido.

Dentro del FFM, el factor Amabilidad y Responsabilidad, dados sus elementos operativos, deberían predecir una menor diversión y un mayor rechazo hacia contenidos humorísticos "negativos", tales como el humor de denigración, negro, etc. Este dato no se ha investigado.

En el estudio que a continuación se presenta, se explora la relación entre el sistema PEN de Eysenck, el modelo de los cinco grandes de McCrae y Costa, y la apreciación del humor. Para llevar a cabo el estudio se empleará la Escala de Apreciación del Humor, EAHU (Carretero-Dios, 2005), basada parcialmente en la propuesta de Willibald Ruch, y que tiene en cuenta seis tipos de humor (humor con incongruencia-resolución; humor sin sentido; humor sexual; humor negro; humor de denigración del hombre; $y$ humor de denigración de la mujer). Se intentarán corroborar los hallazgos hasta ahora encontrados sobre la relación entre personalidad y apreciación del humor, lo que aportaría evidencias externas de validez a la EAHU. 
Con los nuevos contenidos que aporta la EAHU, se estudiarán las nuevas predicciones efectuadas sobre la relación entre las dimensiones básicas de personalidad tratadas, y la diversión y el rechazo mostrados hacia dichos contenidos.

\section{MÉTODO}

\section{Participantes}

La muestra estuvo compuesta por un total de 323 participantes, con una edad media de 31,18 y una desviación tipica de 13,83 (MÍnimo=18; Máximo=76). Del total muestral, 177 eran hombres $(54,8 \%)$, con una edad media de 33,85 años y una desviación típica de 14,93 ; y 146 mujeres $(45,2 \%)$, con una edad media de 27,96 y una desviación típica de 11,65. Del total muestral, 180 participantes eran estudiantes universitarios de la Universidad de Granada, y 143 no estudiantes universitarios con heterogeneidad en cuanto a ocupación laboral y nivel de estudios.

\section{Instrumentos}

Inventario de Personalidad NEO Revisado (NEO-PI-R) de Costa y McCrae (1992). La versión de la adaptación española, realizada por la editorial TEA ediciones (Costa y McCrae, 1999), es similar a la escala original. Cuenta con un total de 60 ítems con una escala de respuesta tipo likert de 5 alternativas que va desde $A=$ "En total desacuerdo"; a $E$ $=$ "Totalmente de acuerdo". Cada uno de los 5 factores que se evalúan (Neuroticismo; Extraversión; Apertura a la Experiencia; Amabilidad y Responsabilidad) se ven representados por 12 ítems.

Inventario de Personalidad de Eysenck Revisado, EPQ-R (Eysenck, Eysenck y Barret, 1985). Instrumento de evaluación que sirve para recoger el modelo de personalidad de los tres grandes factores del sistema PEN de Eysenck; Neuroticismo; Extraversión y Psicoticismo. La versión española (Aguilar, Tous y Pueyo, 1990) cuenta con un total de 100 ítems con una escala de respuesta de verdadero o falso (23 para extraversión, 24 para neuroticismo, 32 para psicoticismo y 21 para la escala de sinceridad). 
Escala de Apreciación del Humor, EAHU (Carretero-Dios, 2005). Escala de 32 items agrupados en seis factores aislados empiricamente, los cuales corresponden a otros tantos tipos de humor: humor con incongruencia-resolución; humor sin sentido; humor sexual; humor negro; humor de denigración del hombre y humor de denigración de la mujer. Cada uno de los factores están representados por un total de seis ítems, excepto para el humor de denigración del hombre y la mujer, con cuatro ítems cada uno. En todos los casos los ítems consisten en chistes escritos o viñetas gráficas, ante los cuales se debe responder según el grado de diversión y rechazo experimentado. Para cada ítem se usan dos escalas tipo Likert de cinco puntos que irian "0" (Nada divertido / Ningún rechazo) hasta "4" (Muy Divertido / Fuerte Rechazo). Cada una de las personas evaluadas obtendría un total de doce puntuaciones parciales, correspondientes a sus totales en diversión y rechazo para cada uno de los tipos de humor, a la vez que dos puntuaciones globales, una para diversión y otra para rechazo, resultado de la suma de las puntuaciones obtenidas para cada componente.

Durante el desarrollo del instrumento se han constatado las propiedades métricas adecuadas de los items y la estabilidad e invarianza factorial a través de muestras distintas. Los valores alfa de Cronbach oscilaron, según el componente de la EAHU, entre 0,69 y 0,82 para la escala de diversión, y entre 0,73 y 0,89 para la de rechazo.

Los cuestionarios aparecian configurando un cuaderno en el que éstos eran presentados en el siguiente orden: EPQ-R, NEO-PI-R y EAHU.

\section{Procedimiento}

Para la recogida de la muestra se usaron dos tipos muestreo no probabilísticos. El primero de ellos, y con la intención de que la muestra no fuera exclusivamente de estudiantes de psicología, consistió en un muestreo incidental llevado a cabo en algunas bibliotecas de la Universidad de Granada. Para éste primer tipo de muestreo se usó siempre el mismo encuestador previamente entrenado. A aquellas personas que accedian a colaborar se les entregaba el cuadernillo de cuestionarios precedido por unas instrucciones estándar. Junto a este 
procedimiento de recogida de muestra, se usó un muestreo por cuotas, siendo éstas el sexo (igual número de hombres que de mujeres), y la edad (mayores de treinta años). Estas cuotas se fijaron teniendo en cuenta los resultados de la investigación que ponen de manifiesto la relevancia del sexo y la edad en la apreciación del humor (Ruch, et al., 1990), de ahí el hecho de recurrir a este segundo procedimiento de muestreo, para tener una muestra con un intervalo amplio de edad, y homogénea en cuanto al número de hombres y mujeres. Para realizar este segundo muestreo se recurrió a un procedimiento de "bola de nieve". El cuadernillo de cuestionarios era el mismo que el usado para el muestreo incidental. El tiempo medio en contestar a las pruebas fue de tres cuartos de hora.

\section{RESULTADOS}

En la tabla 1., pueden observarse las correlaciones parciales (controlando el efecto de la edad y el sexo) y correlaciones parciales corregidas por atenuación, entre cada una de las dimensiones básicas de personalidad y los componentes de la EAHU. Al valorar los resultados, nótese que debido al tamaño muestral, correlaciones bajas (de $r=$ 0,11 a $r=0,15$ ) son marcadas como significativas a una $p<0,05$.

Para la dimensión básica Neuroticismo se puede apreciar como aparece una correlación parcial positiva con la puntuación total en rechazo de la EAHU, tanto si se considera el EPQ-R $(r=0,17, p<$ $0,01$, siendo la atenuada de $r=0,19)$, como el NEO-PI-R, aunque para este caso el valor es muy bajo $(r=0,11, p<0,05$, siendo la atenuada de $r=0,13$ ). Con la puntuación total de diversión no aparece significación estadística.

Siguiendo con el factor neuroticismo, en el caso de los datos obtenidos a través del EPQ-R, se puede apreciar como para todos los componentes de la EAHU, y cuando se consideran las respuestas de rechazo, la correlación parcial es positiva, aunque de forma significativa aparece para la categoría de contenido sexual $(r=0,17, p<0,01$, siendo la atenuada de $r=0,19)$, humor negro $(r=0,16, p<0,01$, siendo la atenuada de $r=0,17)$, y de denigración de la mujer $(r=0,18, p<$ 0,001 , siendo la atenuada de $r=0,22$ ). Para este último componente, las respuestas de diversión se correlacionan negativamente con 
neuroticismo de una forma significativa, aunque de forma bastante baja $(r=-0,11, p<0,05$, siendo la atenuada de $r=-0,13)$. Si los datos a analizar son los obtenidos a través del NEO-PI-R, la tendencia se mantiene para las respuestas de rechazo y diversión, siendo no obstante los valores de correlación más bajos. La correlación parcial positiva más elevada se encuentra con la categoría de humor negro ( $r$ $=0,14, p<0,05$, siendo la atenuada de $r=0,16)$, apareciendo también la significación estadística en el caso de la categoria sexual $(r=0,11$, $p<0,05$, siendo la atenuada de $r=0,12$ ). Al contrario que con la escala de neuroticismo del EPQ-R, con el NEO-PI-R no aparecen más datos de correlación parcial significativos.

Teniendo en cuenta la dimensión básica de Extraversión (tabla 1), aparece una correlación parcial positiva con la puntuación total en diversión de la EAHU, tanto a través del EPQ-R $(r=0,13, p<0,05$, siendo la atenuada de $r=0,14)$, como del NEO-PI-R, siendo de nuevo para este caso los valores más moderados $(r=0,11, p<0,05$, siendo la atenuada de $r=0,13$ ). Con la puntuación total en rechazo, la tendencia es a una correlación parcial negativa, siendo ésta significativa para el EPQ-R $(r=-0,15, p<0,05$, siendo la atenuada de $r=-0,18)$, aunque no para el NEO-PI-R.

De las correlaciones obtenidas entre extraversión y las respuestas de diversión a cada una de los componentes de la EAHU, los valores más elevados aparecen para la dimensión sexual (con el EPQ-R, $r$ $=0,15, p<0,05$, siendo la atenuada de $r=0,19$; con el NEO-PI- $R_{1} r$ $=0,12, p<0,05$, siendo la atenuada de $r=0,14$ ) y de denigración del hombre en el caso del EPQ-R $(r=0,15, p<0,05$, siendo la atenuada de $r=0,20$ ). Además, entre extraversión y las respuestas de rechazo hacia dimensión sexual, aparece una correlación negativa más alta que las observadas hasta ahora usando el EPQ-R $(r=-0,23, p<$ 0,001 , siendo la atenuada de $r=-0,28$ ). Si el test es el NEO-PI-R, aunque también existe significación estadística, el valor es muy bajo $(r=-0,11, p<0,05$, siendo la atenuada de $r=-0,12)$. También, aunque con valores muy bajos y sólo si se tiene en cuenta el EPQ-R, existe una correlación parcial significativa entre extraversión y el rechazo a la categoría de denigración del hombre $(, r=-0,15, p<0,05$, siendo la atenuada de $r=0,20)$, y humor sin sentido $(r=-0,11, p<0,05$, siendo la atenuada de $r=-0,12)$. 
$\stackrel{5}{5}$

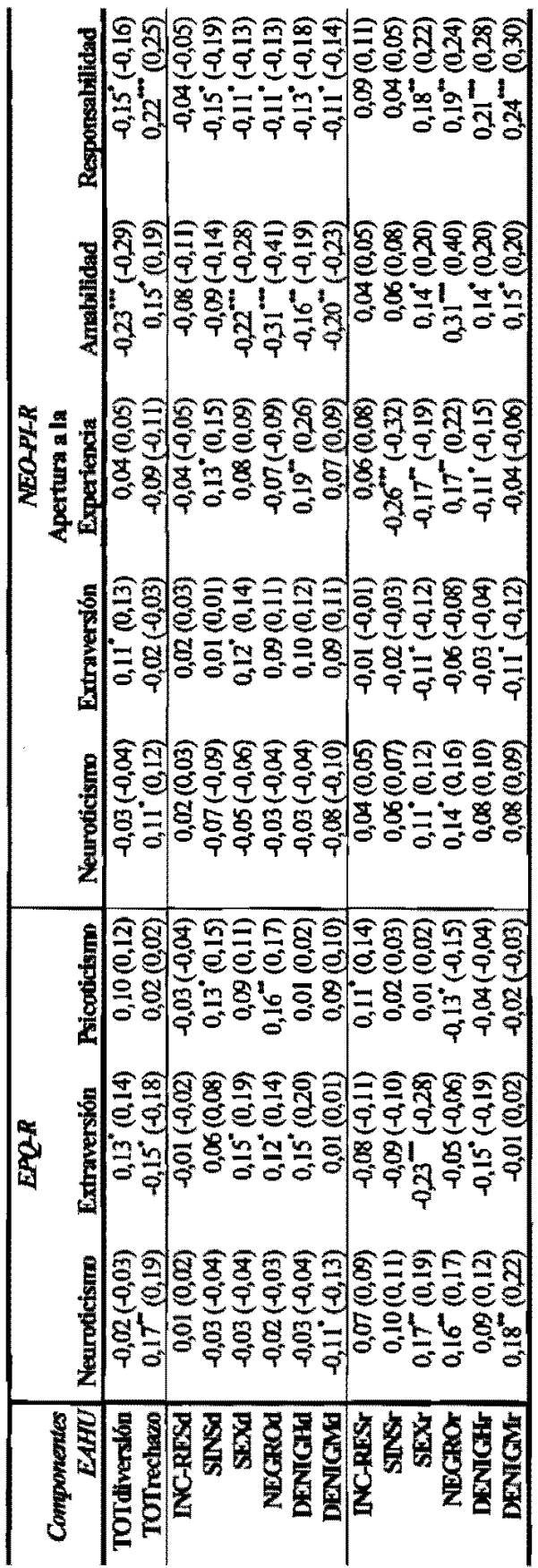

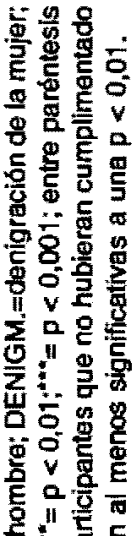

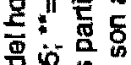

옹음 웜

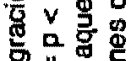

홀"

के

공동

T竞

产察

崖 II N

穴语

홀

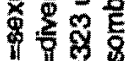

离各里

บั)

8 응

เิ

응

동

8

y 8

密

S 8

का

28

y

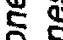

ธิ์

कृ

बे

ชิ

$\div$ 过

to

5

흐등

응

돈

क要㝵

등 훙

if "1 8

능

๘등 흥

通象

은

总量

눈응 당

용

동

동용

형웡

. ᄃ 8

他㔖

山出要

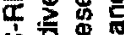

ㅇํㅇ옹 
Para el factor Psicoticismo del EPQ-R, puede observarse una ausencia de significación estadística en las correlaciones parciales calculadas entre éste y la puntuación total en diversión y rechazo. Inspeccionando cada componente de la EAHU, habría que destacar el valor de correlación parcial encontrado para la categoría de humor negro (para las respuestas de diversión, $r=0,16, p<0,01$, siendo la atenuada de $r=0,17$; para las respuestas de rechazo, $r=-0,13, p<$ 0,05 , siendo la atenuada de $r=-0,15$ ). De igual forma, se observa como aparece una correlación positiva entre psicoticismo y la diversión hacia el humor sin sentido $(r=0,13, p<0,05$, siendo la atenuada de $r$ $=0,15$ ), a la vez que con el rechazo hacia el humor con incongruencia resolución $(r=0,11, p<0,05$, siendo la atenuada de $r=0,14)$.

De las correlaciones encontradas entre la dimensión Apertura a la Experiencia del NEO-PI-R y la EAHU, cabría destacar que considerando tanto las puntuaciones totales en diversión como en rechazo, los valores están entorno a cero. Sin embargo, al tener en cuenta cada uno de los componentes de la EAHU puede destacarse como aparece una correlación positiva significativa con la diversión hacia el humor sin sentido $(r=0,13, p<0,05$, siendo la atenuada de $r=0,15)$, a la vez que una negativa con el rechazo hacia el mismo tipo de humor $(r=-0,26$, $p<0,001$, siendo la atenuada de $r=-0,32$ ). De igual forma, aparece una correlación parcial positiva significativa entre apertura a la experiencia y la diversión hacia el humor donde se denigra al hombre ( $r=0,19, p<0,01$, siendo la atenuada de $r=0,26$ ), junto con una negativa con el rechazo hacia el mismo tipo de humor $(r=-0,11, p<$ 0,05 , siendo la atenuada de $r=-0,15$ ). Siguiendo con las respuestas de rechazo, también aparecen correlaciones parciales significativas entre apertura a la experiencia y el humor sexual $(r=-0,17, p<0,001$, siendo la atenuada de $r=-0,19)$ y humor negro $(r=0,17, p<0,001$, siendo la atenuada de $r=0,22$ ), que como puede observarse presentan signo distinto.

Para el caso de la Amabilidad, existe una correlación significativa entre este factor y la puntuación total en diversión $(r=-0,23, p<0,001$, siendo la atenuada $r=-0,29)$, y en rechazo $(r=0,15, p<0,05$, siendo la atenuada $r=0,19$ ), aunque como puede observarse de sigo opuesto. El análisis de cada componente de la EAHU revela que estas correlaciones encontradas para los totales son debidas a la influencia 
de las dimensiones de contenido de la EAHU, ya que erı ningún caso, para las dimensiones estructurales de incongruencia-resolución o sin sentido aparece un dato significativo. Como se puede observar en la tabla 1., las correlaciones parciales entre la amabilidad y la diversión hacia todas las dimensiones de contenido son negativas $y$ significativas. La correlación menor es de $r=-0,16$, para la categoría de denigración del hombre $(p<0,01$, con una correlación corregida por atenuación $r=-0,19$ ), oscilando el resto entre $r=-0,20$ (denigración de la mujer, con una correlación corregida por atenuación de $r=-0,23$ ) y $r=-0,31$ (humor negro, con una correlación corregida por atenuación de $r=-0,41$ ). De igual forma, las puntuaciones en rechazo de todas las categorías de contenido se correlacionan significativamente con el factor amabilidad, pero ahora de manera positiva, y siendo de nuevo el valor más alto el encontrado para la categoría de humor negro ( $r=$ $0,31, p<0,001$, siendo la atenuada de $r=0,40$ ).

Por último, para el factor Responsabilidad, hay que decir que la tendencia es similar a la encontrada para amabilidad. Aparece una correlación significativa entre este factor $y$ el total en diversión $(r=-$ $0,15, p<0,001$, siendo la atenuada $r=-0,16)$, y en rechazo $(r=0,22$, $p<0,001$, siendo la atenuada $r=0,25$ ). Si se tiene en cuenta los componentes de la EAHU, de nuevo se pone de manifiesto, y tal y como aparecía con amabilidad, una correlación negativa estadísticamente significativa entre responsabilidad y la diversión hacia cualquiera de las categorías de contenido, a la vez que correlaciones significativas, pero ahora positivas, entre este factor y el rechazo hacia dichas dimensiones de contenido (véase tabla 1.), siendo éstas últimas más elevadas. No obstante, aparece un dato diferencial en cuanto al patrón encontrado para amabilidad, y es la correlación negativa y significativa encontrada entre responsabilidad y diversión como respuesta al humor $\sin$ sentido $(r=-0,15, p<0,05$, siendo la atenuada de $r=-0,19$ ).

A continuación se presentan análisis de regresión múltiple por pasos sucesivos, considerando a todas las dimensiones de personalidad como variables predictoras, y a cada componente de la EAHU como criterio. Sólo se efectúa este cálculo si existe un patrón significativo de predicción entre personalidad y el componente en cuestión de la EAHU, calculado a través de análisis de regresión simultáneos. 
Para las dos categorías cognitivas de la EAHU (incongruenciaresolución y sin sentido), el único componente para el que la personalidad configura un modelo de predicción significativo según el análisis de regresión simultáneo, $F(8,315)=2,09, p<0,05$, es el que recoge el rechazo hacia el humor sin sentido. Este modelo presenta una $\mathbf{R}=0,28$, con un coeficiente de determinación corregido de $\mathbf{R}^{2}=$ 0,06 . El análisis de regresión múltiple por pasos sucesivos, teniendo como criterio las respuestas de rechazo hacia el humor sin sentido (SINS), puede observarse en la tabla 2. Tal y como puede apreciarse, sólo una variable entra en el único modelo de regresión significativo resultante, y es la apertura a la experiencia. El coeficiente de regresión tipificado es igual a - 0,25 , y dado que sólo entre en el modelo una variable, el control del efecto del resto de variables predictoras (correlación semiparcial), resulta en una correlación de igual valor $(r=$ $-0,25)$. La apertura a la experiencia explica un $4 \%$ de la varianza total de la variable criterio.

Tabla 2. Análisis de regresión múltiple por pasos sucesivos teniendo como variables predictoras las dimensiones de personalidad y como criterio el rechazo hacia el humor sin sentido (SINSr)

\begin{tabular}{ccccccccc}
\hline & $\mathbf{R}$ & $\mathbf{R}^{2} \mathbf{c}$ & $\mathbf{B}$ & Beta & $\mathbf{t}$ & Sig. & $\begin{array}{c}\text { Correlación } \\
\text { parcial } \\
\text { semiparcial }\end{array}$ \\
\hline $\begin{array}{c}\text { Modelo I } \\
\text { A. Experiencia NEO-PI-R }\end{array}$ & 0,25 & 0,04 & $-0,15$ & $-0,25$ & $-3,35$ & 0,001 & $-0,25$ & $-0,25$ \\
\hline
\end{tabular}

Criterio: Rechazo Humor Sin Sentido. $R^{2} c=$ coeficiente de determinación corregido.

Para las respuestas de diversión hacia el humor sexual, el análisis de regresión simultáneo proporciona un modelo significativo, $F(8,315)=$ $3,81, p<0,001$, para el que $R=0,36$ y el coeficiente de determinación corregido es $R^{2}=0,10$. El análisis de regresión múltiple por pasos sucesivos resulta en dos modelos significativos que se pueden observar en la tabla 3. En el modelo 1, la variable introducida es amabilidad, capaz de explicar el $8 \%$ de la varianza total de la diversión hacia el humor sexual, y con un coeficiente de correlación semiparcial de $r=-0,29$. En el segundo modelo se añade la variable extraversión evaluada con el NEO-PI-R, con un coeficiente de regresión tipificado de $0,17, y$ haciendo que la varianza total explicada se incremente levemente $(10 \%)$. 
Tabla 3. Análisis de regresión múltiple por pasos sucesivos teniendo como variables predictoras las dimensiones de personalidad y como criterio la diversión hacia el humor sexual (SEXd)

\begin{tabular}{|c|c|c|c|c|c|c|c|c|}
\hline & \multirow[b]{2}{*}{$\mathbf{R}$} & \multirow[b]{2}{*}{$\mathbf{R}^{1} \mathbf{c}$} & \multirow[b]{2}{*}{ B } & \multirow[b]{2}{*}{ Beta } & & \multirow[b]{2}{*}{ Sig. } & \multicolumn{2}{|c|}{ Correlación } \\
\hline & & & & & & & parcial & semiparcial \\
\hline $\begin{array}{c}\text { Madelo } 1 \\
\text { Amabilidad NEO-PI-R }\end{array}$ & 0,29 & 0,08 & $-0,26$ & $-0,29$ & $-4,37$ & 0,000 & $-0,29$ & $-0,29$ \\
\hline $\begin{array}{c}\text { Madelo 2 } \\
\text { Amabilidad NEO-PI-R } \\
\text { Extraversión NEO-PJ-R }\end{array}$ & 0,33 & 0,10 & $\begin{array}{r}-0,29 \\
0,13\end{array}$ & $\begin{array}{r}-0,32 \\
0,17\end{array}$ & $\begin{array}{r}-4,75 \\
2,40\end{array}$ & $\begin{array}{l}0,000 \\
0,013\end{array}$ & $\begin{array}{r}-0,32 \\
0,17\end{array}$ & $\begin{array}{r}-0,31 \\
0,16\end{array}$ \\
\hline
\end{tabular}

Criterio: Diversión Humor Sexual. $\mathbf{R}^{2} \mathrm{C}=$ coeficiente de determinación corregido

El análisis de regresión simultáneo con todas las variables de personalidad como predictoras, y el rechazo hacia el humor sexual como criterio, revela un efecto significativo de la personalidad sobre éste, $F(8,315)=3,85, p<0,001$, siendo $R=0,35$, y $R^{2}$ corregida 0,11 . En la tabla 4, se presentan los datos resultantes del análisis de regresión por pasos sucesivos.

Tabla 4. Análisis de regresión múltiple por pasos sucesivos teniendo como variables predictoras las dimensiones de personalidad y como criterio el rechazo hacia el humor sexual (SEXr)

\begin{tabular}{|c|c|c|c|c|c|c|c|c|}
\hline & \multirow[b]{2}{*}{$\mathbf{R}$} & \multirow[b]{2}{*}{$\mathbf{R}^{2} \mathbf{c}$} & \multirow[b]{2}{*}{ B } & \multirow[b]{2}{*}{ Beta } & \multirow[b]{2}{*}{$t$} & \multirow[b]{2}{*}{ Sig. } & \multicolumn{2}{|c|}{ Correlación } \\
\hline & & & & & & & parcial & semiparcial \\
\hline $\begin{array}{l}\text { Modelo } 1 \\
\text { Neuroticismo EPQ-R }\end{array}$ & 0,24 & 0,05 & 0,26 & 0,24 & 3,64 & 0,000 & 0,24 & 0,24 \\
\hline $\begin{array}{l}\text { Modelo } 2 \\
\text { Neuroticismo EPQ-R } \\
\text { Amabilidad NEO-PI-R }\end{array}$ & 0,29 & 0,08 & $\begin{array}{l}0,29 \\
0,19\end{array}$ & $\begin{array}{l}0,26 \\
0,18\end{array}$ & $\begin{array}{l}3,81 \\
2,76\end{array}$ & $\begin{array}{l}0,000 \\
0,009\end{array}$ & $\begin{array}{l}0,25 \\
0,17\end{array}$ & $\begin{array}{l}0,25 \\
0,17\end{array}$ \\
\hline $\begin{array}{c}\text { Madelo } 3 \\
\text { Neuroticismo EPQ-R } \\
\text { Amabilidad NEO-PI-R } \\
\text { Responssbilidad NEO-PI-R }\end{array}$ & 0,31 & 0,09 & $\begin{array}{l}0,28 \\
0,14 \\
0,12\end{array}$ & $\begin{array}{l}0,25 \\
0,15 \\
0,13\end{array}$ & $\begin{array}{l}3,75 \\
2,23 \\
1,96\end{array}$ & $\begin{array}{l}0,000 \\
0,024 \\
0,051\end{array}$ & $\begin{array}{l}0,25 \\
0,15 \\
0,13\end{array}$ & $\begin{array}{l}0,24 \\
0,14 \\
0,12\end{array}$ \\
\hline
\end{tabular}

Criterio: Rechazo Humor Sexual. $\mathbf{R}^{2} \mathrm{c}=$ coeficiente de determinación corregido

Los datos de la tabla 4, ponen de manifiesto tres modelos de regresión significativos. En el modelo 1, la variable introducida es neuroticismo evaluada con el EPQ-R, que explica el $5 \%$ de la varianza total del rechazo hacia el humor sexual, y que presenta un coeficiente de correlación semiparcial y tipificado de 0,24. El segundo modelo, que llega a explicar el $8 \%$ de la varianza total del criterio, incorpora la variable amabilidad, con un coeficiente de regresión tipificado de 0,18 , 
frente al 0,26 que ahora refleja neuroticismo. El tercer y último modelo, explica el $9 \%$ de la varianza total del criterio, e incorpora la variable responsabilidad, con un coeficiente de regresión tipificado de 0,13 , frente al 0,15 del factor amabilidad, y el 0,25 de neuroticismo. Los coeficientes de correlación parcial y semiparcial de los tres modelos sirven para poner de manifiesto que el factor neuroticismo es el que realiza una mayor aportación a $\mathrm{R}^{2}$.

Al considerar las respuestas de diversión hacia el humor negro, de nuevo el análisis de regresión simultáneo resulta en un efecto significativo de las variables de personalidad como predictoras de la diversión hacia este tipo de humor, $F(8,315)=7,90, p<0,001$, siendo $R=0,46$, y $R^{2} c=0,19$. El análisis de regresión múltiple por pasos sucesivos, que se presenta en la tabla 5., facilita un total de dos modelos de regresión significativos. En el modelo 1, la variable introducida es amabilidad, capaz de explicar el $15 \%$ de la varianza total de la diversión hacia el humor negro, y con un coeficiente de correlación parcial y semiparcial de $r=-0,40$. En el segundo modelo, que pasa a explicar el $43 \%$ de la varianza total, se incorpora la variable neuroticismo evaluada con el EPQ-R, con un coeficiente de regresión tipificado de $-0,15$. La inspección de las correlaciones parcial y semiparcial sirve para concluir que la variable amabilidad es la que aporta más a $R^{2}$. Sobre los factores de personalidad excluidos hay que señalar que los coeficientes más elevados aparecen para el factor psicoticismo, con una correlación parcial $r=0,08$, y con un coeficiente de regresión estandarizado, en caso de haber sido introducido en el modelo, de Beta $=0,07$.

Tabla 5. Análisis de regresión múltiple por pasos sucesivos teniendo como variables predictoras las dimensiones de personalidad y como criterio la diversión hacia el humor negro (NEGROd)

\begin{tabular}{ccccccccc}
\hline & R & R'c & B & Beta & t & Sig & parcial & semiparcial \\
\hline $\begin{array}{c}\text { Modelo I } \\
\text { Amabilidad NEO-PI-R }\end{array}$ & 0,40 & 0,15 & -38 & $-0,40$ & $-6,38$ & 0,000 & $-0,40$ & $-0,40$ \\
\hline $\begin{array}{c}\text { Modelo 2 } \\
\text { Amabilidad NEO-PI-R }\end{array}$ & 0,43 & 0,18 & $-0,40$ & $-0,42$ & $-6,74$ & 0,000 & $-0,42$ & $-0,41$ \\
Neuroticismo EPQ-R & & & $-0,16$ & $-0,15$ & $-2,42$ & 0,017 & $-0,16$ & $-0,15$ \\
\hline
\end{tabular}

Criterio: Diversión Humor Negro. $R^{2} \mathrm{c}=$ coeficiente de determinación corregido 
El análisis de regresión simultáneo con todas las variables de personalidad como predictoras, y el rechazo hacia el humor negro como criterio, resulta en un efecto significativo, $F(8,315)=5,91, p<$ 0,001 , donde $R=0,43, y R^{2}$ corregida $=0,16$. A continuación se presenta la tabla 6., donde aparecen los datos relevantes derivados del análisis de regresión por pasos sucesivos teniendo como criterio al rechazo hacia el humor negro.

En la tabla 6., se pueden observar un total de tres modelos de regresión significativos. En el modelo 1, el cual explica el 10\% de la varianza total del criterio, la variable introducida es amabilidad, con un coeficiente de correlación semiparcial y tipificado de $r=0,32$. En el segundo modelo, donde se pasa a explicar el $12 \%$ de la varianza total del criterio, se añade la variable neuroticismo evaluada por el EPQ-R, con un coeficiente de regresión tipificado de 0,17 , frente al 0,34 que en este modelo presenta el factor amabilidad. El último modelo significativo explica el $15 \%$ de la varianza total del criterio, y donde se incorpora la apertura a la experiencia, con un coeficiente de regresión tipificado de 0,17 , frente al 0,18 del factor neuroticismo, y al 0,31 de amabilidad. Los coeficientes de correlación parcial y semiparcial de los modelos resultantes servirian para concluir que el factor amabilidad es el que realiza una mayor aportación a $\mathrm{R}^{2}$.

Tabla 6. Análisis de regresión múltiple por pasos sucesivos teniendo como variables predictoras las dimensiones de personalidad y como criterio el rechazo hacia el humor negro (NEGROr)

\begin{tabular}{|c|c|c|c|c|c|c|c|c|}
\hline & \multirow[b]{2}{*}{$\mathbf{R}$} & \multirow[b]{2}{*}{$\mathbf{R}^{2} \mathbf{c}$} & \multirow[b]{2}{*}{ B } & \multirow[b]{2}{*}{ Beta } & \multirow[b]{2}{*}{$\mathbf{t}$} & \multirow[b]{2}{*}{ Sig. } & \multicolumn{2}{|c|}{ Correlación } \\
\hline & & & & & & & parcial & semiparcial \\
\hline $\begin{array}{c}\text { Modelo } 1 \\
\text { Amabilidad NEO-PI-R }\end{array}$ & 0,33 & 0,10 & 0,32 & 0,33 & 4,95 & 0,000 & 0,32 & 0,32 \\
\hline $\begin{array}{c}\text { Modelo } 2 \\
\text { Amabilidad NEO-PI-R } \\
\text { Neuroticismo EPQ-R }\end{array}$ & 0,37 & 0,12 & $\begin{array}{l}0,34 \\
0,18\end{array}$ & $\begin{array}{l}0,34 \\
0,17\end{array}$ & $\begin{array}{l}5,34 \\
2,61\end{array}$ & $\begin{array}{l}0,000 \\
0,010\end{array}$ & $\begin{array}{l}0,34 \\
0,17\end{array}$ & $\begin{array}{l}0,34 \\
0,16\end{array}$ \\
\hline $\begin{array}{c}\text { Modelo 3 } \\
\text { Amabilidad NEO-PI-R } \\
\text { Neuroticismo EPQ-R } \\
\text { A. Experiencia NEO-PI-R }\end{array}$ & 0,40 & 0,15 & $\begin{array}{l}0,31 \\
0,19 \\
0,18 \\
\end{array}$ & $\begin{array}{l}0,31 \\
0,18 \\
0,17\end{array}$ & $\begin{array}{l}4,88 \\
2,81 \\
2,74 \\
\end{array}$ & $\begin{array}{l}0,000 \\
0,005 \\
0,007\end{array}$ & $\begin{array}{l}0,32 \\
0,19 \\
0,18\end{array}$ & $\begin{array}{l}0,31 \\
0,18 \\
0,17\end{array}$ \\
\hline
\end{tabular}

Criterio: Rechazo Humor Negro. $R^{2} c=$ coeficiente de determinación corregido

Un modelo de predicción significativo según el análisis de regresión simultáneo, surge al considerar como criterio las respuestas de diversión hacia el humor de denigración del hombre, $F(8,315)=3,20$, 
$p<0,01$. Este modelo presenta una $R=0,33$, con un coeficiente de determinación corregido de $\mathrm{R}^{2}=0,09$. $\mathrm{E}$ análisis de regresión múltiple por pasos sucesivos, resulta en un total de tres modelos de regresión significativos (tabla 7). El primer modelo, que viene a explicar un $4 \%$ de la varianza total del criterio, recoge al factor apertura a la experiencia, con un coeficiente de regresión tipificado, y correlación parcial y semiparcial de 0,22 . En el segundo modelo se incluye el factor amabilidad, y se pasa a explicar un $7 \%$ de la varianza del criterio. El factor amabilidad aparece con un coeficiente de regresión tipificado de $-0,19$, que es igual que su coeficiente de correlación parcial. En este segundo modelo, la apertura a la experiencia presente un valor de Beta $=0,23$. El tercer y último modelo de regresión significativo explica un $8 \%$ de la varianza del criterio. La variable que se incorpora es responsabilidad, con Beta $=-0,14$, siendo este valor para amabilidad de $-0,16$, y para apertura a la experiencia de 0,24 .

TABLA 7. Análisis de regresión múltiple por pasos sucesivos teniendo como variables predictoras las dimensiones de personalidad y como criterio la diversión hacla el humor de denigración del hombre (DENIGHd)

\begin{tabular}{|c|c|c|c|c|c|c|c|c|}
\hline & \multirow[b]{2}{*}{$\mathbf{R}$} & \multirow[b]{2}{*}{$\mathbf{R}^{2} \mathbf{c}$} & \multirow[b]{2}{*}{ B } & \multirow[b]{2}{*}{ Beta } & \multirow[b]{2}{*}{$t$} & \multirow[b]{2}{*}{ Sig } & \multicolumn{2}{|c|}{ Correlación } \\
\hline & & & & & & & parcial & semipareial \\
\hline $\begin{array}{c}\text { Modelo 1 } \\
\text { A. Experiencia NEO-PI-R }\end{array}$ & 0,22 & 0,04 & 0,17 & 0,22 & 4,95 & 0,003 & 0,22 & 0,22 \\
\hline $\begin{array}{c}\text { Modelo 2 } \\
\text { A. Experiencia NEO-PI-R } \\
\text { Amabilidad NEO-PI-R }\end{array}$ & 0,27 & 0,07 & $\begin{array}{r}0,16 \\
-0,12\end{array}$ & $\begin{array}{r}0,23 \\
-0,19\end{array}$ & $\begin{array}{r}3,51 \\
-2,79\end{array}$ & $\begin{array}{l}0,001 \\
0,006\end{array}$ & $\begin{array}{r}0,23 \\
-0,19\end{array}$ & $\begin{array}{r}0,23 \\
-0,18\end{array}$ \\
\hline $\begin{array}{c}\text { Modelo } 3 \\
\text { A. Experiencla NEO-PI-R } \\
\text { Amabilidad NEO-PI-R } \\
\text { Responsabilidad NEQ-PI-R }\end{array}$ & 0,30 & 0,08 & $\begin{array}{r}0,15 \\
-0,12 \\
-0,10\end{array}$ & $\begin{array}{r}0,24 \\
-0,16 \\
-0,14\end{array}$ & $\begin{array}{r}3,57 \\
-2,34 \\
-1,94\end{array}$ & $\begin{array}{l}0,000 \\
0,005 \\
0,057\end{array}$ & $\begin{array}{r}0,24 \\
-0,16 \\
-0,13\end{array}$ & $\begin{array}{r}0,23 \\
-0,15 \\
-0,12\end{array}$ \\
\hline
\end{tabular}

Criterio: Diversión Humor Denigración Hombre. $R^{2} c=$ coeficiente de determinación corregido

El análisis de regresión simultáneo con todas las variables de personalidad como predictoras, y el rechazo hacia el humor de denigración del hombre como criterio, revela un efecto significativo, $F(8,315)=2,66, p<0,01$, con una $R=0,31$, y $R^{2}$ corregida de 0,08 .

Los datos del análisis de regresión por pasos sucesivos teniendo como criterio el rechazo hacia el humor de denigración del hombre (tabla 8), ponen de manifiesto la presencia de un solo modelo de 
regresión significativo, que explica el $4 \%$ de la varianza total del criterio, y que está conformado por el factor responsabilidad, para el que el coeficiente de regresión tipificado es de 0,19. Al observar las variables excluidas, destacan el factor extraversión y neuroticismo del EPQ-R, además de la dimensión amabilidad del NEO-PI-R. La correlación parcial para extraversión y neuroticismo es $r=-0,11$ y $r=0,11$ respectivamente, con un valor de Beta igual al de correlación parcial en ambos casos. La correlación parcial indicada para amabilidad es de $r=0,10$, con Beta $=0,09$.

Tabla 8. Análisis de regresión múltiple por pasos sucesivos teniendo como variables predictoras las dimensiones de personalidad y como criterio el rechazo hacia el humor de denigración del hombre (DENIGHr)

\begin{tabular}{ccccccccc}
\hline & $\mathbf{R}$ & $\mathbf{R}^{1} \mathbf{c}$ & $\mathbf{B}$ & Beta & t & Sig. & $\begin{array}{c}\text { Correlación } \\
\text { parcial } \\
\text { semiparcial }\end{array}$ \\
\hline $\begin{array}{c}\text { Modelo I } \\
\text { Responsabilldad NEO-PI-R }\end{array}$ & 0,19 & 0,04 & 0,14 & 0,19 & 2,82 & 0,005 & 0,19 & 0,19 \\
\hline
\end{tabular}

Criterio: Rechazo Humor Denigración Hombre. $R^{2} \mathrm{C}=$ coeflciente de determinación corregido.

Al aplicar el análisis de regresión simultáneo, considerando a los factores de personalidad como variables predictoras, y a la diversión hacia la última dimensión de contenido de la EAHU que queda por analizar, denigración de la mujer, como criterio, se pone de manifiesto un efecto significativo, $F(8,315)=4,89, p<0,001$, con una $R=0,40, y$ $R^{2}$ corregida de 0,14 . El análisis de regresión múltiple por pasos sucesivos, proporciona un total de tres modelos de regresión significativos (Tabla 9.). En el primer modelo, el cual explica un $8 \%$ de la varianza total del criterio, aparece el factor amabilidad, con un coeficiente de regresión tipificado de $-0,29$. En el segundo modelo, donde se explica el $12 \%$ de la varianza total del criterio, se incorpora el factor responsabilidad, con un coeficiente de regresión tipificado de 0,20 , y un coeficiente de regresión parcial y semiparcial de $r=-0,21$ y $r=-0,20$ respectivamente. En este segundo modelo, la amabilidad presente un coeficiente Beta, de correlación parcial y semiparcial de 0,30 . En el último modelo de regresión significativo, se pasa a explicar un $13 \%$ de la varianza total del criterio. La variable incluida es el factor 
neuroticismo del NEO-PI-R, con Beta $=-0,15$. En este tercer modelo, la amabilidad presenta un coeficiente de regresión tipificado de $-0,27$, y la responsabilidad de $-0,21$.

Tabla 9. Análisis de regresión múltiple por pasos sucesivos teniendo como variables predictoras las dimensiones de personalidad y como criterio la diversion hacia el humor de denigración de la mujer(DENIGMd)

\begin{tabular}{|c|c|c|c|c|c|c|c|c|}
\hline & \multirow[b]{2}{*}{$\mathbf{R}$} & \multirow[b]{2}{*}{$\mathbf{R}^{1} \mathbf{c}$} & \multirow[b]{2}{*}{$\mathbf{B}$} & \multirow[b]{2}{*}{ Beta } & \multirow[b]{2}{*}{ t } & \multirow[b]{2}{*}{ Sig } & \multicolumn{2}{|c|}{ Correlación } \\
\hline & & & & & & & parcial & semiparcial \\
\hline $\begin{array}{c}\text { Madelo I } \\
\text { Amabilidad NEO-PJ-R }\end{array}$ & 0,29 & 0,08 & $-0,20$ & $-0,29$ & $-3,45$ & 0,000 & $-0,29$ & $-0,29$ \\
\hline $\begin{array}{c}\text { Modelo } 2 \\
\text { Amabilidad NEO-PI-R } \\
\text { Responsabilidad NEO-PI-R }\end{array}$ & 0,35 & 0,12 & $\begin{array}{r}-0,20 \\
-0,15\end{array}$ & $\begin{array}{r}-0,30 \\
-0,20\end{array}$ & $\begin{array}{l}-4,66 \\
-3,17\end{array}$ & $\begin{array}{l}0,000 \\
0,002\end{array}$ & $\begin{array}{l}-0,30 \\
-0,21\end{array}$ & $\begin{array}{r}-0,30 \\
-0,20\end{array}$ \\
\hline $\begin{array}{c}\text { Modelo 3 } \\
\text { Amabilidad NEO-PI-R } \\
\text { Responsabilidad NEO-PI-R } \\
\text { Neuroticismo NEO-PI-R }\end{array}$ & 0,38 & 0,13 & $\begin{array}{l}-0,18 \\
-0,16 \\
-0,11\end{array}$ & $\begin{array}{r}-0,27 \\
-0,21 \\
-0,15\end{array}$ & $\begin{array}{l}4,14 \\
-3,34 \\
-2,15\end{array}$ & $\begin{array}{l}0,000 \\
0,001 \\
0,033\end{array}$ & $\begin{array}{l}-0,27 \\
-0,22 \\
-0,15\end{array}$ & $\begin{array}{l}-0,26 \\
-0,21 \\
-0,14\end{array}$ \\
\hline
\end{tabular}

Crlterlo: Dlversión Humor Denlgración Mujer. $\mathrm{R}^{2} \mathrm{c}=$ coeflclente de determlnación corregldo

Al tener en cuenta las respuestas de rechazo hacia el humor de denigración de la mujer como criterio del análisis de regresión simultáneo, de nuevo aparece un efecto significativo de las variables de personalidad como predictoras, $F(8,315)=7,06, p<0,001$, siendo $R=0,46, y R^{2}$ corregida $=0,18$. El análisis de regresión múltiple por pasos sucesivos, que se presenta en la tabla 10,, facilita un total de cinco modelos de regresión significativos.

En el modelo 1 (tabla 10), la variable introducida es responsabilidad, capaz de explicar el $6 \%$ de la varianza total del criterio, y con un coeficiente de regresión tipificado, de correlación parcial y semiparcial de $r=0,26$. El segundo modelo, el cual explica el $11 \%$ de la varianza total del rechazo hacia el humor de denigración de la mujer, incorpora la variable amabilidad, con un coeficiente de regresión tipificado de 0,24 , siendo el de responsabilidad de 0,25. El tercer modelo de regresión significativo explica un $16 \%$ de la varianza total del criterio y añade el factor neuroticismo del EPQ-R, para el que Beta es de 0,23, pasando ese valor para el factor amabilidad a 0,27 , y para el factor responsabilidad a 0,20 . El siguiente modelo de regresión explica el $17 \%$ de la varianza total del criterio, e incorpora el factor extraversión 
del NEO-PI-R, con un valor negativo del coeficiente de regresión tipificado (Beta $=-0,14$ ). Los valores Beta de los factores de este cuarto modelo disminuyen para el caso de responsabilidad (Beta $=0,18$ ) y neuroticismo (Beta $=0,21$ ), aumentando el de amabilidad $(0,30)$. El quinto y último modelo de regresión significativo explica un $19 \%$ de la varianza total del rechazo hacia el humor de denigración de la mujer, e incorpora la dimensión apertura a la experiencia con un valor Beta = 0,14 . La evaluación de las correlaciones parciales y semiparciales de los cinco modelos de regresión, vendría a poner de manifiesto que la variable amabilidad es la que provoca un mayor aumento en $\mathrm{R}^{2}$. Sobre los factores de personalidad excluidos, el coeficiente de correlación parcial más elevado es para el factor psicoticismo, $r=-0,09$, el cual presenta un coeficiente de regresión tipificado en caso de haber sido introducido en el modelo de Beta $=-0,08$.

Tabla 10. Análisis de regresión múltiple por pasos sucesivos teniendo como variables predictoras las dimensiones de personalidad y como criterio el rechazo hacia el humor de denigración de la mujer (DENIGMr)

\begin{tabular}{|c|c|c|c|c|c|c|c|c|}
\hline & \multirow[b]{2}{*}{$\mathbf{R}$} & \multirow[b]{2}{*}{$\mathbf{R}^{2} \mathbf{c}$} & \multirow[b]{2}{*}{ B } & \multirow[b]{2}{*}{ Beta } & \multirow[b]{2}{*}{$\mathbf{t}$} & \multirow[b]{2}{*}{ Sig. } & \multicolumn{2}{|c|}{ Correlación } \\
\hline & & & & & & & parcial & semiparcial \\
\hline $\begin{array}{c}\text { Modelo I } \\
\text { Responsabilidad NEO-PI-R }\end{array}$ & 0,26 & 0,06 & 0,20 & 0,26 & 3,86 & 0,000 & 0,26 & 0,26 \\
\hline $\begin{array}{c}\text { Modelo } 2 \\
\text { Responsabilidad NEO-PI-R } \\
\text { Amabilidad NEO-PI-R }\end{array}$ & 0,35 & 0,11 & $\begin{array}{l}0,19 \\
0,21\end{array}$ & $\begin{array}{l}0,25 \\
0,24\end{array}$ & $\begin{array}{l}3,96 \\
3,73\end{array}$ & $\begin{array}{l}0,000 \\
0,000\end{array}$ & $\begin{array}{l}0,26 \\
0,25\end{array}$ & $\begin{array}{l}0,25 \\
0,24\end{array}$ \\
\hline Modelo 3 & 0,41 & 0,16 & & & & & & \\
\hline Responsabilidad NEO-PI-R & & & 0,16 & 0,20 & 3,16 & 0,002 & 0,21 & 0,20 \\
\hline Amabilidad NEO-PI-R & & & 0,24 & 0,27 & 4,31 & 0,000 & 0,28 & 0,27 \\
\hline Neuroticismo EPQ-R & & & 0,19 & 0,23 & 3,50 & 0,001 & 0,23 & 0,22 \\
\hline Modelo 4 & 0,43 & 0,17 & & & & & & \\
\hline Responsabilidad NEO-PI-R & & & 0,14 & 0,18 & 2,82 & 0,005 & 0,19 & 0,18 \\
\hline Amabilidad NEO-PI-R & & & 0,27 & 0,30 & 4,68 & 0,000 & 0,30 & 0,29 \\
\hline Neuroticismo EPQ-R & & & 0,18 & 0,21 & 3,29 & 0,001 & 0,22 & 0,20 \\
\hline Extraversión NEO-PI-R & & & $-0,09$ & $-0,14$ & 2,11 & 0,036 & $-0,14$ & $-0,13$ \\
\hline Modelo 5 & 0,45 & 0,19 & & & & & & \\
\hline Responsabilidad NEO-PI-R & & & 0,16 & 0,21 & 3,19 & 0,002 & 0,21 & 0,20 \\
\hline Amabilidad NEO-PI-R & & & 0,26 & 0,29 & 4,57 & 0,000 & 0,30 & 0,28 \\
\hline Neuroticismo EPQ-R & & & 0,22 & 0,26 & 3,83 & 0,000 & 0,26 & 0,24 \\
\hline Extraversión NEO-PI-R & & & $-0,10$ & $-0,14$ & 2,24 & 0,026 & $-0,15$ & $-0,14$ \\
\hline A. Experiencia NEO-PI-R & & & 0,28 & 0,14 & 2,09 & 0,037 & 0,14 & 0,13 \\
\hline
\end{tabular}

Criterio: Rechazo Humor Denigración Mujer. $\mathrm{R}^{2} \mathrm{c}=$ coeficiente de determinación corregido 


\section{DISCusión}

En el estudio que se acaba de presentar se ha analizado la relación entre las dimensiones de la apreciación del humor recogidas en la EAHU y las dimensiones básicas de personalidad consideradas en el EPQ-R y en el NEO-PI-R. A continuación se discuten los hallazgos más importantes de este estudio, y se reflexiona acerca de la repercusión de éstos sobre las evidencias externas de validez de la EAHU.

No puede olvidarse que con la EAHU se consideran dos tipos de respuesta, una de valencia positiva (diversión), y otra de valencia negativa (rechazo), y que vendrian a ocupar el espacio de los aspectos emocionales de la apreciación del humor. Dentro de las dimensiones básicas de personalidad, aquellas más vinculadas a estos aspectos serían la extraversión (valencia positiva) y el neuroticismo (valencia negativa). Un análisis de las correlaciones parciales, controlando la influencia de la edad y el sexo, entre las puntuaciones totales de diversión y rechazo obtenidas con la EAHU, y las dimensiones básicas de personalidad, ha revelado que la dimensión básica de extraversión, tanto evaluada a través del EPQ-R, como del NEO-PI-R, se muestra correlacionada de una manera positiva principalmente con las respuestas de diversión, y negativamente, aunque con valores más bajos, con las respuestas de rechazo. Es decir, las personas más extravertidas se divierten generalmente más con los componentes de la EAHU, a la vez que muestran un menor rechazo hacia éstos. Si ahora, en cambio, se considera el factor neuroticismo, los resultados de estas correlaciones van en la dirección opuesta, es decir, una correlación positiva más elevada con la respuesta de rechazo, y negativa, aunque inferior, con la puntuación total en diversión. Estos hallazgos suponen una confirmación de las predicciones realizadas.

Aunque los resultados anteriores son bastante claros en cuanto a la tendencia aparecida, conviene hacer dos matizaciones. La primera es que las correlaciones son bajas, y aún pudiendo aparecer significación estadística, los valores más elevados, obtenidos a través del EPQ-R, son de $r=0,17$, entre neuroticismo y rechazo total $(p<$ $0,01)$, y $r=-0,15$, entre extraversión y rechazo total $(p<0,05)$. La segunda matización es que estos resultados surgen, como se ha dicho, 
al tener en cuenta los totales en diversión y rechazo. Cuando el análisis es con cada componente de la EAHU por separado, la aportación principal es debida a las categorías de contenido y no a las cognitivas de la EAHU.

Respecto a las correlaciones bajas, habría que señalar que los datos de investigación, tanto teniendo como referencia al sistema PEN como al FFM, estarían en consonancia con lo que aquí se ha encontrado (Ruch, 1992; Ruch y Hehl, 1998). No obstante, estos valores bajos de correlación, y a pesar de la falta de significación estadistica para la extraversión y el neuroticismo, no deben descartar la relevancia de éstos en la investigación teórica sobre la apreciación del humor. La tendencia encontrada es bastante clara, y además, cuando se ha trabajado con factores no básicos pero relacionados con los generales de neuroticismo y extraversión, las correlaciones son más elevadas y muestran significación estadística en la dirección esperada (Ruch y Köhler, 1998). Así, los valores de correlación entre neuroticismo, extraversión y apreciación del humor son normales al tratarse la apreciación de un chiste o viñeta de una respuesta mucho menos estable y dependiente de eventos contextuales que la de los factores básicos considerados, lo que hace que la predicción sea leve (Pelechano, 2000; Pelechano y Servando, 2004).

En cuanto al segundo matiz anteriormente señalado, y relacionado con el hecho de que los valores de las correlaciones obtenidos con los totales de la EAHU son debidos a la aportación de las dimensiones de contenido, habría que resaltar que se trata de un hecho igualmente encontrado en otros trabajos donde el contenido estudiado es el sexual, (Ruch, 1992), y que se trataría además de un dato con explicación teórica.

Las categorías cognitivas de la EAHU se ocuparian de recoger fundamentalmente los aspectos cognitivos vinculados con el constructo apreciación del humor. Estos aspectos cognitivos se relacionan con la comprensión, percepción o preferencia por las incongruencias simples (humor con incongruencia-resolución) frente a las complejas y no completamente resueltas (humor sin sentido). De esta forma, los factores de personalidad a considerar, en cuanto a que se espere una relación más estrecha de éstos con dichos cornponentes, serian los que están más afectados igualmente por estos aspectos cognitivos, y no el neuroticismo y la extraversión, que 
se vinculan a aspectos fundamentalmente emocionales. Además, estos factores presentan otros marcadores, como el elevado interés por el sexo, despreocupación, osadía, etc., para el caso de la extraversión; o el miedo a la evaluación, culpabilidad, tensión, emotividad, para el caso del neuroticismo. Estos marcadores hacen a arnbos factores más importantes para predecir las puntuaciones en las categorías de contenido recogidas en la EAHU. Así, con las categorías de contenido como la sexual, humor negro o de denigración, las relaciones si deberían ser más elevadas, en cuanto a una mayor diversión para extraversión, y un mayor rechazo para neuroticismo. Pues bien, los datos encontrados en el estudio aquí presentado para las categorías cognitivas y para las de contenido, apoyan estos supuestos. No obstante, a continuación se discuten con más detalle estos hallazgos.

En cuanto a los componentes cognitivos, hay que decir que de las dimensiones básicas de personalidad evaluadas, éstos están más presentes en el factor de apertura a la experiencia evaluado por el NEO-PI-R, y en menor medida en el factor psicoticismo. La apertura a la experiencia se compone fundamentalmente por facetas referentes a la curiosidad, imaginación, inteligencia y creatividad, y el psicoticismo incorpora ciertos elementos de creatividad. Esto lleva a plantear que sean factores importantes, en especial el primero, para apresar los aspectos cognitivos de la apreciación del humor. En concreto se esperaba (Ruch, 1994; Ruch y Hehl, 1998) que los participantes con puntuaciones altas en estos constructos se diviertan más con el humor sin sentido, a la vez que lo rechazan menos. Las correlaciones parciales obtenidas para este estudio apoyarian lo predicho, especialmente en el caso de la apertura a la experiencia, donde se logra la significación estadística en la dirección predicha, mientras que para el psicoticismo, como en investigaciones anteriores (Ruch y Hehl, 1998), las correlaciones son más débiles. Junto a esto hay que decir que al aplicar los análisis de regresión por pasos sucesivos considerando a las dimensiones básicas de personalidad como predictores, y como criterio las categorías estructurales de la EAHU, la única dimensión predictora incluida en los modelos de regresión es la apertura a la experiencia, pero siempre y cuando se considere el rechazo hacia el humor sin sentido como criterio. 
Para el humor con incongruencia-resolución, las correlaciones han sido en todos los casos no significativas, y las ecuaciones de regresión no proporcionan ningún modelo de predicción significativo para este componente. No obstante, se observa tanto para psicoticismo como para apertura a la experiencia, una tendencia opuesta a la aparecida al considerar el humor sin sentido. Es decir, ahora las correlaciones negativas aparecen con la diversión hacia este tipo de humor, y las positivas con el rechazo. Esta tendencia coincide con los resultados del grupo de Ruch, aunque en este caso los valores son más bajos. Sin embargo, habría que recordar que el polo bajo de apertura a la experiencia o psicoticismo no revela un gusto por lo simple, o intolerancia a lo novedoso o nuevas experiencias. Para recoger estos aspectos habría que trabajar con otras medidas de constructos tales como la intolerancia a la incertidumbre, donde puntuaciones elevadas revelasen una evitación de lo nuevo o incierto. En estos casos si deberían aparecer relaciones significativas con la categoría estructural incongruencia-resolución, por su más estrecha conexión entre los dos elementos puestos en relación.

$\mathrm{Si}$ el análisis se centra en los aspectos motivacionales, primero habría que destacar, tal y como anteriormente se ha señalado, que la extraversión y neuroticismo, han mostrado correlaciones positivas con la diversión hacia las categorías de contenido para el primer caso, e igualmente positivas con el rechazo hacia esas dimensiones, en el segundo, siendo estas correlaciones más elevadas que la que se han obtenido al correlacionar estos factores con el humor con incongruenciaresolución y humor sin sentido. El neuroticismo suele aparecer en los modelos significativos de predicción, pero a continuación de los factores que se muestran más estrechamente unidos con cada categoría de contenido de la EAHU como criterio, y que son fundamentalmente la amabilidad y la responsabilidad. Así, el neuroticismo suele aparecer dentro de los modelos de regresión que tienen al rechazo hacia las dimensiones de contenido como criterio. La extraversión, mientras tanto, ha aparecido dentro de los modelos de regresión que tenían como variable criterio la diversión hacia el humor sexual, pero no en el resto. Este dato sirve para apoyar las predicciones efectuadas.

Para recoger los aspectos motivacionales de la apreciación del humor, es importante contar con escalas validadas con contenidos 
humorísticos diversos. Sin embargo, dentro del campo de estudio de la apreciación del humor esto no está presente. Los datos se reducen al contenido sexual, y relaciones como las que aqui se han estudiado con factores empíricamente aislados de humor negro o de denigración son una excepción.

Teniendo en cuenta los resultados encontrados para el factor amabilidad y responsabilidad del NEO-PI-R, la primera observación iría dirigida a señalar como los valores de correlación son los más altos que se han encontrado en comparación con los aparecidos para el resto de dimensiones básicas de personalidad. Tanto la amabilidad como la responsabilidad muestran correlaciones positivas y significativas con el rechazo hacia los contenidos humorísticos que recoge la EAHU, y negativas con la diversión hacia los mismos contenidos. Además, ambos factores suelen estar integrados en los modelos de regresión significativos como los predictores más importantes de los contenidos humorísticos.

Con un factor como el de amabilidad (Agreeableness), caracterizado por rasgos como la simpatía, sensibilidad hacia los demás, etc., los datos encontrados son los esperados. De igual forma, los resultados para el factor responsabilidad, con su faceta vinculada al "sentido del deber", muy relacionada con principios éticos y morales, vendrían a suponer igualmente un apoyo teórico a las relaciones lógicas a establecer con los componentes de contenido de la EAHU. Estos hallazgos que se acaban de indicar no habían sido investigados con instrumentos psicométricamente avalados. No obstante, y por lo comentado, las relaciones encontradas irían en la dirección de lo esperado. Además, estos dos factores, amabilidad y responsabilidad, muestran relaciones más altas que las aparecidas para los anteriormente discutidos neuroticismo y extraversión. Siguiendo el modelo de Parámetros del profesor Vicente Pelechano, el cual ha sido tomado como referencia para el desarrollo de esta investigación, aparte de por su elementos definicionales que los hacen especialmente importantes para las dimensiones de contenido como las aquí tratadas, las correlaciones más elevadas podrían reflejar el hecho de tratares de dimensiones que no tienen el respaldo suficiente como para entenderlas como temperamentales básicas en cuanto a estabilidad y consolidación (Pelechano, 1996; Pelechano y Servando, 2004). Se 
podrian entender como factores no básicos de la personalidad, y con un nivel de estabilidad y consolidación más vinculado a contextos de vida, lo que provocaría correlaciones más elevadas con la apreciación del humor. Esto se podría poner en relación con los resultados encontrados para el factor psicoticismo.

La dimensión Psicoticismo propuesta por Eysenck, que recogería facetas como la socialización y empatía llegando a la hostilidad, agresividad, ha mostrado correlaciones significativas con el humor negro en la dirección esperada, es decir, una correlación positiva con la diversión y negativa con el rechazo. Sin embargo, los valores son inferiores a los aparecidos para amabilidad y responsabilidad, no incluyéndose además en los modelos de regresión significativos. Así, estos datos más moderados, dado el contenido relevante de psicoticismo por su esperada vinculación con las categorías de contenido de la EAHU, podrían servir de apoyo a la hipótesis sobre la importancia de tener en cuenta la diferencia de estabilidad y consolidación de las variables puestas en relación, a pesar de poder compartir marcadores definicionales, dado el nivel más básico de la dimensión psicoticismo (Pelechano y Servando, 2004) con respecto a amabilidad y responsabilidad.

Siguiendo con los predictores más importantes de los aspectos motivacionales de la apreciación del humor, es decir, con las preferencias hacia contenidos determinados, habría que señalar la relación entre el factor apertura a la experiencia, y especialmente las respuestas hacia el humor de denigración del hombre, de la mujer, y el humor sexual. A través de las correlaciones se observa como existe una relación positiva entre la apertura a la experiencia y la diversión hacia estos tipos de humor, a la vez que una negativa con el rechazo. Para el caso de la denigración del hombre, los datos alcanzan la significación estadística, y los análisis de regresión lo señalan como el predictor más importante de las respuestas de diversión hacia este tipo de humor, aunque para el rechazo no aparece en ningún modelo de regresión significativo. A su vez, y con respecto al humor sexual, aparece una correlación negativa significativa entre apertura a la experiencia y rechazo hacia el humor sexual, siendo la correlación con diversión no relevante. Para el humor de denigración de la mujer, aunque se observa la misma tendencia que la aparecida para el humor 
sexual y de denigración del hombre, no se llega a alcanzar la significación estadística.

Como se ha dicho, el factor de apertura a la experiencia se ve delimitado por marcadores como la fantasía, curiosidad, o imaginación, que lo hacían especialmente relevante para la categoría humorística del humor sin sentido. Sin embargo, igualmente se ve compuesto por elementos definicionales más relacionados con tener una "mente abierta", ser tolerante y estar abierto a experiencias variadas. Los datos de investigación del grupo de Ruch han puesto de manifiesto como los valores opuestos a una mentalidad abierta, y más representativos de una preferencia por lo establecido, o un rechazo por la variedad y el cambio, están negativamente relacionados con la diversión hacia el humor sexual (Forabosco y Ruch, 1994). Asi, los componentes definicionales de la apertura a la experiencia irían en un sentido contrario, sirviendo para recoger en cierta forma el polo opuesto al conservadurismo y a la mentalidad cerrada. Por ello, los datos encontrados para la apertura a la experiencia serían esperables teóricamente hablando.

A pesar de lo recién comentado, la relación entre el factor apertura a la experiencia y la categoría de humor negro es opuesta a las encontradas con el resto de categorías de contenido. Para este caso la correlación es negativa entre apertura a la experiencia y diversión, y positiva con el rechazo, aunque sólo significativa para este último caso. De igual forma, para estas respuestas de rechazo, la apertura a la experiencia forma parte de los modelos de regresión significativos.

Las facetas de la apertura a la experiencia vinculadas a la tolerancia, aceptación de la variedad y de lo nuevo, favorecería a que se mostrase una mayor inclinación por contenidos humorísticos "delicados", como el sexual, o el que se basa en caricaturizar características de los demás (humor de denigración). Sin embargo, si el humor versa sobre un hecho trágico, o se hace a costa del daño físico o psíquico real ajeno, la mayor empatía y sensibilidad de las personas con puntuaciones altas en apertura a la experiencia, provocaría que este tipo de humor se viera como menos divertido, a la vez que es más rechazado. Sería como una preferencia por lo diferente y fuera de lo establecido, pero siempre y cuando esto no sea a costa del daño real de los demás. Es decir, tolerancia y apertura de ideas, pero con límites. 
Llegados a este momento, y habiendo agrupado los resultados encontrados en función de su aportación para recoger los aspectos cognitivos, emocionales o motivacionales de la apreciación del humor, se podría concluir que los datos van en la dirección de las relaciones predichas entre personalidad y los componentes de la apreciación del humor recogidos en la EAHU, y que por lo tanto proporcionan evidencias positivas de validez externa de las puntuaciones obtenidas con dicha escala. No obstante, los siguientes estudios de validez externa deberán ir enriqueciendo las aportaciones, y el componente estructural incongruencia-resolución requerirá un análisis más cuidadoso teniendo en cuenta sus componentes definicionales.

En este estudio, y con las distintas variables predictoras con las que se ha trabajado, la mayoría de los valores significativos de las correlaciones parciales (controlando la edad y el sexo), o de las correlaciones parciales de las ecuaciones de regresión donde se controlaba el efecto del resto de variables incluidas en el modelo, se han situado entorno a $r=0,20$ o incluso menos. Los valores de correlación encontrados significarían, por un lado, y lo que es lógico, que otras variables no tenidas en cuenta deben estar influyendo sobre la respuesta de apreciación, y por otro, que dado el nivel básico de las dimensiones predictoras, la predicción es la esperada. Futuras investigaciones deben trabajar con niveles de estabilidad distintos de una misma dimensión o varias, y explorar las diferencias predictivas, algo que ya desde el modelo de parámetros, propuesta de referencia para este trabajo, se ha contrastado en varias ocasiones (Pastor, 2004; Pelechano, 1996; Pelechano y De Miguel, 2000).

\section{REFERENCIAS BIBLIOGRÁFICAS}

Aguilar, A., Tous, J. M., y Pueyo, A. A. (1990). Adaptación y estudio psicométrico del EPQ-R. Anuario de Psicologia, 46, 101-118.

Carretero-Dios, H. (2005). Sentido del Humor: construcción de la Escala de Apreciación del Humor, EAHU. Tesis doctoral: Universidad de Granada.

Eysenck, H. J. (1942). The appreciation of humor: An experimental and theoretical study. British Journal of Psychology, 32, 295-309. Eysenck, S., Eysenck, H. J., y Barrett, P. (1985). A revised version of 
the psychoticism scale. Personality and Individual Difference, 6, 21-29.

Forabosco, G. (1992). Cognitive aspects of the humor process: The concept of incongruity. Humor: International Journal of Humor Research, 5, 45-68.

Forabosco, G., y Ruch, W. (1994). Sensation seeking, social attitudes, and humor appreciation in Italy. Personality and Individual Differences, 16, 515-528.

Lefcourt, H. (2002). Humor. En C.R. Snyder y S. Lopez (eds.), Handbook of Positive psychology (pp. 619-631). London: Oxford University Press.

Martin, R.A. (1998). Approaches to the sense of humor: a historical review. En W. Ruch (ed.), The sense of humor: Explorations of a personality characteristic (pp. 15-62). Berlin: Mouton de Gruyter. McCrae, R. R., y Costa, P. T. Jr. (1999). A five-factor theory of personality. In L.A. Pervin y O.P. John (eds.), Handbook of personality: theory and research (2nd ed.) (pp. 139-153). New York: Guilford Press.

Norrick, N. R. (2003). On humor. Humor: International Journal of Humor Research, 16, 426-429.

Pastor, A. (2004). Trastornos de personalidad y dimensiones de personalidad: una aproximación desde el modelo de parámetros. Tesis doctoral: Universidad de la Laguna.

Pelechano, V. (1996). Psicologia de la personalidad I. Teorias. Barcelona: Ariel.

Pelechano, V. (2000). Psicología sistemática de la personalidad. Barcelona: Ariel.

Pelechano, V. y De Miguel, A. (2000). Estabilidad y cambio evolutivo/ generacional en personalidad. Análisis y Modificación de Conducta, 26, 817-854.

Pelechano, V., y Rivero, A. J. (2004). Una contrastación parcial del modelo de sabiduría de Berlín: resultados, reflexión y una propuesta. Análisis y Modificación de Conducta, 30, 465-493.

Pelechano, V., y Servando, M. A. (2004). ¿Qué es la personalidad? Madrid: Biblioteca Nueva.

Roeckelein, J. (2002). The Psychology of Humor: A reference guide and annotated bibliography. London: Greenwood Press. 
Ruch, W. (1992). Assessment of appreciation of humor: Studies with the 3 WD humor test. En C.D. Spielberger y J.M. Butcher (eds.), Advances in personality assessment, (pp. 27-75). Hillsdale, NJ: Erlbaum.

Ruch, W. (1994). Temperament, Eysenck's PEN system, and humor-related traits. Humor: Intemational Journal of Humor Research, $7,209-244$.

Ruch, W. (1998). Sense of humor: A new look at an old concept. En W. Ruch (ed.), The sense of humor: Explorations of a personality characteristic (pp. 3-14). Berlin: Mouton de Gruyter.

Ruch, W. (1999). The sense of nonsense lies in the nonsense of sense. Comment on Paolillo's (1998) Gary Larsen's Far Side: Nonsense? Nonsense! Humor: International Journal of Humor Research, 12, 71-93.

Ruch, W., y Carrell, A. (1998). Trait cheerfulness and the sense of humor. Personality and Individual Differences, 24, 551-558.

Ruch, W., y Hehl, F.J. (1998). A two-model of humor appreciation: Its relation to aesthetic appreciation and simplicity-complexity of personality. En W. Ruch (ed.), The sense of humor: Explorations of a personality characteristic (pp. 109-142). Berlin: Mouton de Gruyter.

Ruch, W., y Köhler, G. (1998). A temperament approach to humor. En W. Ruch (ed.), The sense of humor: Explorations of a personality characteristic (pp. 203-230). Berlin: Mouton de Gruyter.

Ruch, W., McGhee, P.E., y Hehl, F.J. (1990). Age differences in the enjoyment of incongruity-resolution and nonsense humor during adulthood. Psychology and Aging, 5, 348-355.

Wycoff, E.B., y Prior, B. (2003). Cognitive processing, creativity, apprehension, and the humorous personality. North American Journal of Psychology, 5, 31-44. 